\title{
On the 2D Quantum Tunneling
}

\author{
H. MOHAMmadpour \\ Physics Department, Azarbaijan Shahid Madani University, 53714-161 Tabriz, Iran \\ (Received January 24, 2016; in final form June 27, 2016)
}

\begin{abstract}
In this paper, we have solved a quantum tunneling problem for 2-dimensional systems, including electron gas and graphene. In spite of the one-dimensional scattering problems, in two dimensions, we observe phenomenon of tunneling at energies above the barrier. This effect is an analogue to the total internal reflection in optics. The scattering amplitudes inside the barrier region exhibit decaying behavior corresponding to optical evanescentwave coupling, not only in energies below barrier height, but also above barrier. Velocity-selecting transmission, corresponding to angle-resolved beam filtering effect is one of the achievements of the paper. The famous Hartman effect which occurs normally at sub-barrier energies and has previously been studied for graphene is also addressed. The results manifest occurrence of the Hartman effect for over-barrier energies, as well.
\end{abstract}

DOI: 10.12693/APhysPolA.130.769

PACS/topics: 72.10.-d, 72.10.Bg, 72.80.Vp, 72.90.+y, 73.21.Fg, 73.23.-b, 73.23.Ad, 73.40.Gk

\section{Introduction}

Electron tunneling through barriers is among the most fundamental quantum concepts [1-6]. According to the phenomenon, in the one-dimensional structures as an electron beam with the energy below the barrier height, arriving to the interface with barrier, undergoes evanescent transport through barrier [7], meanwhile, overbarrier transport which is carried by electrons having energy higher than potential barrier, is carried by propagating waves. In two-dimensional (as well as 3D) systems, as well, one observes evanescent transmission at energies below barrier. Although in case of graphene, the Klein tunneling prohibits evanescent transport for normal incidence to the interface.

In contradiction to $1 \mathrm{D}$ case, at $2 \mathrm{D}$ and $3 \mathrm{D}$ structures, we expect evanescent transmission over barrier for some incidence angle interval around the normal to interface $[2,3]$ beyond which one observes propagating waves for energies far below and far above the barrier.

This phenomenon (i.e. over-barrier tunneling) is an electron-wave analogue of the well-known total internal reflection and evanescent-wave coupling in optics. The imaginary wave vector at transport direction which is resultant of the negative contribution of kinetic energy to the total energy at the barrier region is responsible for tunneling. At this paper we first show as reminder that in two-dimensional systems, e.g. two-dimensional electron gas (2DEG) and graphene, at large enough incidence angles, the imaginary wave vector and evanescent transmission emerge at longitudinal direction. The critical angle for a specified potential barrier is determined by the energy. In this paper, velocity-selecting transmission or angle-resolved beam filtering as well as the Hartman effect are studied at this regime [8-10]. (Hint: in 3D media with 1D potential profile, the plane of the incident electron beam direction and the normal to the interface are sustained during the scattering hence makes the third direction irrelevant and the problem reduces to a $2 \mathrm{D}$ one.) Our findings indicate that at over-barrier energy,
1 - the electron transmission occurs mainly at small angles with respect to the normal to interface of the regions and 2 - the Hartmann effect is revealed for incident angles larger than the critical angle for that energy.

\section{Model and results}

At the outset we remind the simplest quantum mechanics problem; electron transport through a barrier in the 1D space. The Hamiltonian of general system satisfies Eq. (1):

$$
H \psi=E \psi, \quad H=T+V(\boldsymbol{r}) .
$$

$T$ is the kinetic energy operator that is written for the Schrödinger electrons as $-\frac{\hbar^{2}}{2 m} \frac{\mathrm{d}^{2}}{\mathrm{~d} x^{2}}$ where $x$ is the coordinate along system, $m$ is effective mass of electron and $V(\boldsymbol{r})=V(x)$ is the potential profile.

In this case for an incident electron with energy $E$ the corresponding wave vector is $k_{x}=k=\sqrt{\frac{2 m E}{\hbar^{2}}}$. In the barrier region with height equal to $V$, one obtains $k^{\prime}{ }_{x}=$ $k^{\prime}=\sqrt{\frac{2 m(E-V)}{\hbar^{2}}}$. Hence, for $E<V, k^{\prime}$ is imaginary and results in evanescent transmission while for $E>V$ we have oscillatory propagation.

Now, let us turn to the case of $2 \mathrm{D}$ systems. For this situation in the general Eq. (1) the kinetic energy, $T$, for electrons in 2DEG is written as $-\frac{\hbar^{2}}{2 m} \nabla^{2}$ and for $2 \mathrm{D}$ monolayer graphene is explained as $-\mathrm{i} \hbar v_{\mathrm{F}} \sigma \nabla$, where $v_{\mathrm{F}}$ and $\boldsymbol{\sigma}$ are respectively the Fermi energy and Pauli matrix in the two sublattices space of graphene. $V(\boldsymbol{r})=V(x, y)$ is the potential profile at plane of electron gas, i.e. $x-y$ plane. For the purpose of this paper, we consider onedimensional barrier profile as $V(x, y)=V ; 0 \leq x \leq L$ and $V(x, y)=0$ otherwise.

Let us consider an electron with energy and incidence angle, $E$ and $\theta$. Inside the barrier region, they take amounts $E-V$ and $\theta^{\prime}$ (Fig. 1).

Due to the translational invariance in $y$ direction, the wave vector of electrons in this direction is conserved by scattering from the barrier, i.e. 

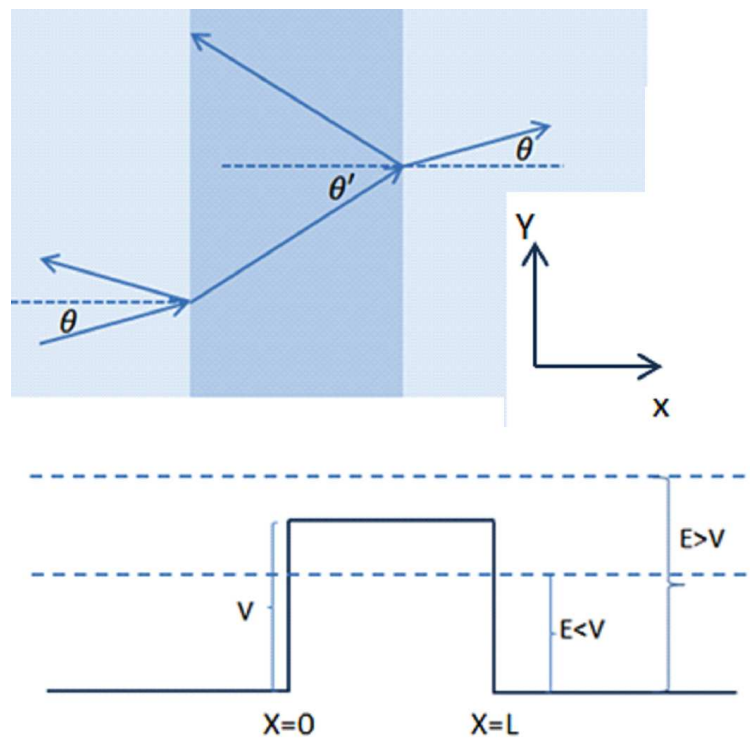

Fig. 1. (top) Schematic of the 2D system in which the middle (dark) region is the barrier layer and electron beams are shown by arrows. An electron incident from the left region to the interface with angle $\theta$ with respect to the normal to interface (dashed lines) is partly reflected to the same region. At the middle region it undergoes first scattering at the second interface as reflection with angle $\theta^{\prime}$ and transmission to the third region with angle $\theta$. (bottom) The 1D barrier energy profile and typical incident electron energy is depicted at two regimes; under-barrier and over-barrier energies.

$$
\begin{aligned}
& k_{y}=k_{y}^{\prime}, \\
& k \sin \theta=k^{\prime} \sin \theta^{\prime} \Longrightarrow \sin \theta^{\prime}=\frac{k}{k^{\prime}} \sin \theta= \\
& \quad \sqrt{\frac{E}{E-V}} \sin \theta .
\end{aligned}
$$

$\theta$ is angle between incidence vector and normal to the interface. Accordingly, in the case of $\frac{k}{k^{\prime}}>1$, we have $\theta^{\prime}>\theta$. So, there is a critical angle,

$$
\theta_{\mathrm{c}}=\arcsin \left(\frac{k^{\prime}}{k}\right)=\arcsin \left(\sqrt{\frac{E-V}{E}}\right),
$$

at which $\theta^{\prime}=\pi / 2$ and above which $\theta^{\prime}$ is imaginary. This corresponds to imaginary $k_{x}^{\prime}$, because $k_{x}^{\prime}=k^{\prime} \cos \theta^{\prime}$.

This phenomenon is described by separating the wave function into the propagating component in the transverse $(y)$ direction, which reduces the problem to a $1 \mathrm{D}$ one with energy effectively reduced by the kinetic energy part related to the propagation in the $y$ direction; hence, an over-barrier transmission in $2 \mathrm{D}$ could be reduced to an under-barrier 1D problem.

Consider a 2DEG in which the barrier of height $V$ is located in $0 \leq x \leq L$. The wave functions at different regions read as

$$
\begin{aligned}
& \psi_{1}(x, y)=\mathrm{e}^{\mathrm{i} k_{x} x+\mathrm{i} k_{y} y}+r \mathrm{e}^{-\mathrm{i} k_{x} x+\mathrm{i} k_{y} y}, \\
& \psi_{2}(x, y)=a \mathrm{e}^{\mathrm{i} k_{x}^{\prime} x+\mathrm{i} k_{y} y}+b \mathrm{e}^{-\mathrm{i} k_{x} x+\mathrm{i} k_{y} y}, \\
& \psi_{3}(x, y)=t \mathrm{e}^{\mathrm{i} k_{x} x+\mathrm{i} k_{y} y} .
\end{aligned}
$$

$\tan (\theta)=k_{y} / k_{x}$ and $\tan \left(\theta^{\prime}\right)=k_{y} / k^{\prime}{ }_{x}$. We impose boundary conditions according to the following equations in order to obtain the scattering amplitudes

$$
\begin{aligned}
& \psi_{1}(x=0)=\psi_{2}(x=0), \frac{\partial \psi_{1}}{\partial x}(x=0)=\frac{\partial \psi_{2}}{\partial x}(x=0), \\
& \psi_{2}(x=L)=\psi_{3}(x=L) \\
& \frac{\partial \psi_{2}}{\partial x}(x=L)=\frac{\partial \psi_{3}}{\partial x}(x=L) .
\end{aligned}
$$

The transmission amplitude, $T(E, \theta)=|t(\theta)|^{2}$ is obtained as

$$
T(E, \theta)=\frac{4 k_{x}^{4}}{4 k_{x 4}^{2} k_{x}^{\prime 2}+\left(k_{x}^{2}-k_{x}^{\prime 2}\right)^{2} \cos ^{2} k_{x}^{\prime 2} L} .
$$

The same situation occurs in the case of graphene as a $2 \mathrm{D}$ electron system, with a difference in the $E-k$ relations, being linear in the case of graphene. This linear relation changes Eqs. (2) and (3) as

$$
\begin{gathered}
\sin \theta^{\prime}=\frac{k}{k^{\prime}} \sin \theta=\frac{E}{E-V} \sin \theta, \quad \theta_{c}= \\
\arcsin \left(\frac{k^{\prime}}{k}\right)=\arcsin \left(\frac{E-V}{E}\right) .
\end{gathered}
$$

Therefore, for the both cases (2DEG and graphene), we expect, according to Eqs. (2), (3) and (7), that for an energy of amount $E>|E-V|$ relation $\theta^{\prime}>\theta$ holds, hence there is a critical incidence angle, determined by Eq. (3) (for graphene by Eq. (7)), above which the amplitudes of transferred waves to the barrier region are descending with the length of this region. This results in elimination of the transmitted waves through the barrier region for large incidence angles in a quantum barrier structure of enough length (the thickness in $x$ direction).

\subsection{Velocity and angle selecting transmission}

The above phenomenon suggests a simple velocityselecting transmission or angle-resolved beam filtering effect, which data are presented in this section.

In Fig. 2, the function $T$ obtained from Eq. (7) for $2 \mathrm{DEG}$, is plot as a function of angle for different lengths, $L$ and different amounts of $V$. All the lengths and energies are scaled respectively by wavelength, $\lambda$, and energy of the incident electron, $E$, that is assumed $1 \mathrm{meV}$.

It is apparent from these diagrams that for electrons of energy $E<|E-V|$, at large $L$, the transmission amplitude is filtered for large angles and only small angles are present at the other side of barrier. In other words, there are just high velocity electrons (in $x$-direction) that are permitted to transmit through the barrier and the slow ones die out midway inside barrier.

Figure 2a gathers the diagrams of transmission amplitude for barriers of thickness $\frac{L}{\lambda}=3$ and different heights. At large thicknesses like this, as we expect, only wavelike propagation survives and evanescent ones are eliminated. Accordingly, we observe pronounced transmission at large angles for small barrier height, i.e., $\frac{V}{E}=0.1$ and by increasing this amount by 0.5 and 0.9 , the transmission is restricted to smaller angle intervals. 

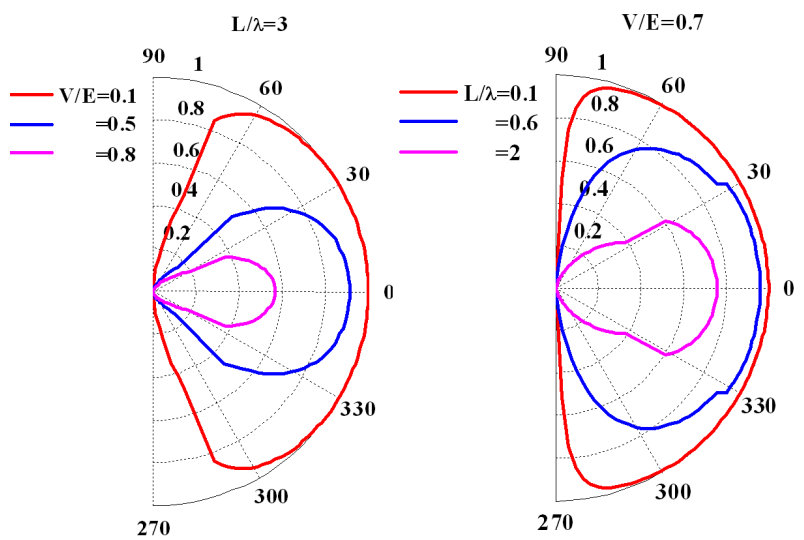

Fig. 2. (a) Angle-resolved transmission amplitude $T(\theta)$ for $\frac{L}{\lambda}=3$ and different potential barrier in terms of the incident energy, i.e. $V / E$. (b) $T(\theta)$ for $\frac{V}{E}=0.7$ and different amounts of $L / \lambda$.

In Fig. 2b the barrier height is hold at $\frac{V}{E}=0.7$ and different thicknesses are employed. It shows that by increasing $\frac{L}{\lambda}$ from (a typical value of) 0.1 to 2 , transmission at large angles is absent.

In the case of graphene, a similar situation occurs for the transmission amplitude calculated in Ref. [2].

Before proceeding to explain results of tunneling studies on graphene, it is worth to mention some points. In Refs. $[11,12]$ the difference between electron transmission in the $2 \mathrm{DEG}$ and graphene has been investigated in detail through the symmetries of transport.

For the symmetric tunneling barrier, it has been shown at [2] that in graphene, for incident electrons of energy below barrier, the system is transparent for normal incidence i.e. $\theta=0$ and this result is independent of the barrier thickness and height. The same result is expected at energies above barrier undoubtedly (to be convinced we note that the over-barrier incidence must be at least as transparent as the under barrier one).

In comparison however it was shown in Fig. 2 that for Schrödinger electrons of 2DEG, the transmission amplitude reduces by increasing the barrier thickness, $\frac{L}{\lambda}$, and its height, $\frac{V}{E}$, even at over-barrier energies. This is on account of the evanescent nature of electrons wave function at the barrier region. This situation occurs also at underbarrier transmission, which is obvious from our quantum mechanics knowledge.

The common result of both cases of Schrödinger electrons of 2DEG and the Dirac fermions of graphene, is that the tunneling footprint is present at over-barrier transmission according to the system characteristics.

In order to study another phenomena related to the over-barrier transmission, at the next section we present our calculations of the transmission time through the barrier in graphene.

\subsection{Hartman effect}

The Hartman effect is another result of the over-barrier tunneling at two dimensions. This effect is related to group delay time $\tau_{\mathrm{g}}$ which is the time delay between the appearance of wavepacket's peak at $x=0$ and $x=L$ for a particle which tunnels through a rectangular barrier at this region. In a symmetric rectangular barrier, the group delay time is given by the energy derivative of the transmission phase shift

$$
\tau_{\mathrm{g}}=\hbar \frac{\mathrm{d}\left(\phi_{\mathrm{t}}+k^{\prime}{ }_{x} L\right)}{\mathrm{d} E},
$$

where $\phi_{\mathrm{t}}$ is the phase of transmitted wave to which we assign zero at the interface between first region and the barrier region (This assumption is common in all scattering problems as was also used in the previous section such that when the boundary condition was applied at the first interface, the total phase at $x=0$ equaled zero.) So the phase that electron wave acquired at the second interface equals to $k_{x}^{\prime} L$.

According to the Hartman effect, which has already been observed at conventional semiconductor structures as well as in monolayer graphene, the group delay time is independent of barrier thickness, $L$.

On the other hand, dwell time is the time spent by a particle in the barrier region, $0<x<L$ regardless of whether it is ultimately transmitted or reflected and is defined as [10]:

$$
\tau_{\mathrm{d}}=\frac{\int_{0}^{L}\left|\psi_{2}(x)\right|^{2} \mathrm{~d} x}{v_{\mathrm{F}} \cos \theta},
$$

where $\psi_{2}(x)$ is the wave function at the barrier region and $v_{\mathrm{F}}$ is the Fermi velocity. It has been shown that in graphene the relation $\tau_{\mathrm{d}}=\tau_{\mathrm{g}}$ holds [10]. So we can check the Hartman effect using Eq. (9). In Ref. [10] this effect was studied in a single barrier and double barrier graphene for energies below the barrier. The results showed that for an incidence angle $\theta$, there is an energy above which the Hartman effect is evident. The authors considered under-barrier energies only. But because of the $2 \mathrm{D}$ nature of the system (that makes $\theta$ nonzero), the transmitted electron wave function at the barrier region can have a longitudinal wave vector $k_{x}^{\prime}$ of either real or imaginary (required condition for the Hartman effect at 2D) types. The condition for occurrence of either of these was discussed at the previous section.

We have depicted the calculated results of $\tau_{\mathrm{g}}$ for a (single barrier) graphene structure in Fig. 3. According to the amounts of parameters used in Eq. (9) for energy (about $1 \mathrm{meV}$ ), and width of the barrier region, the amounts of $\tau_{\mathrm{g}}$ are of picosecond order.

We observe that the Hartman effect is present at overbarrier energies, too.

\section{Conclusion}

The problem of two-dimensional quantum tunneling through a $1 \mathrm{D}$ barrier profile was studied for 2DEG and graphene. At the $2 \mathrm{D}$ systems, electron waves are evanescent not only for energies below the barrier but also at over-barrier energies, too. This effect which corresponds to the total internal reflection and evanescent-wave coupling in optics is utilized in the velocity selective beam 


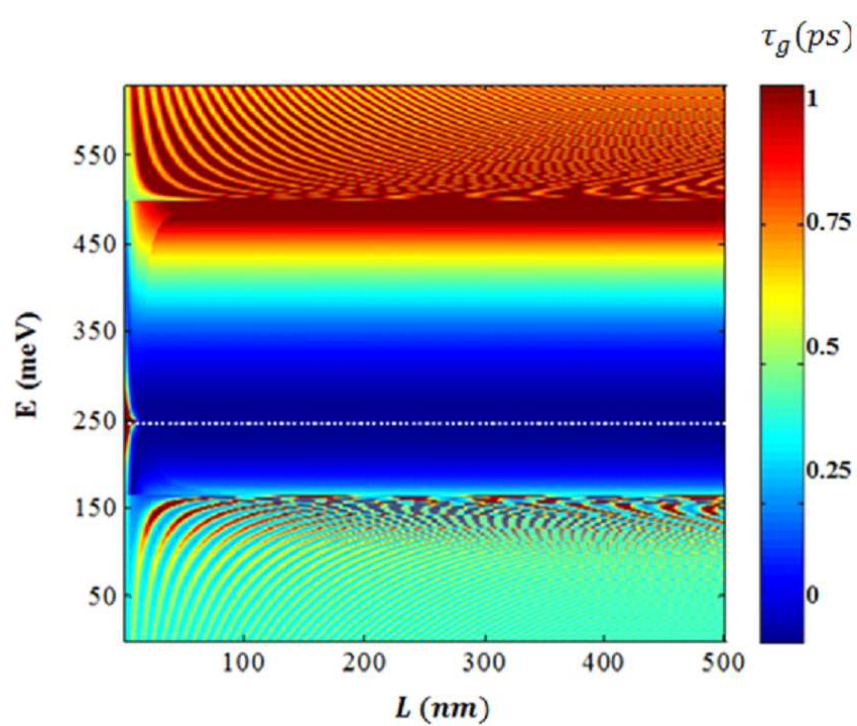

Fig. 3. The tunneling time in ps unit as a function of energy and the barrier thickness in a single barrier of graphene for $\frac{V}{E}=250$ and $\theta=\pi / 6$. The dotted line shows the barrier height.

filtering or angle-resolved transmission as well as in the Hartman effect which holds at under-barrier energies as well.

\section{Acknowledgments}

This paper was supported by Azarbaijan Shahid Madani University.

\section{References}

[1] K.S. Novoselov, A.K. Geim, S.V. Morozov, D. Jiang, Y. Zhang, S.V. Dubonos, I.V. Grigorieva, A.A. Firsov, Science 306, 666 (2004).

[2] M.I. Katsnelson, K.S. Novoselov, A.K. Geim, Nature Phys. 2, 620 (2006).

[3] H. Mohammadpour, A. Asgari, Phys. Lett. A $\mathbf{3 7 5}$, 10 (2011).

[4] Th. Martin, R. Landauer, Phys. Rev. A 45, 2611 (1992).

[5] E.H. Hauge, J.A. Støvneng, Rev. Mod. Phys. 61, 917 (1989).

[6] M. Büttiker, Phys. Rev. B 27, 6178 (1983).

[7] S. Gasiorowicz, Quantum Physics, 3rd ed., John Wiley \& Sons, New York 2003.

[8] T.E. Hartman, J. Appl. Phys. 33, 3427 (1962).

[9] J.R. Fletcher, J. Phys. C 18, L55 (1985).

[10] Z. Wu, K. Chang, J.T. Liu, X.J. Li, K.S. Chan, J. Appl. Phys. 105, 043702 (2009).

[11] M.M. Asmar, S.E. Ulloa, Phys. Rev. B 91, 165407 (2015). 